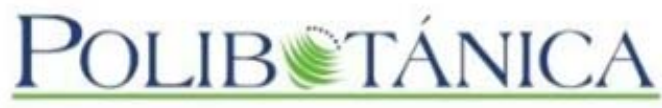

\title{
Polibotánica
}

ISSN electrónico: 2395-9525

polibotanica@gmail.com

Instituto Politécnico Nacional

México

http:www.polibotanica.mx

\section{ANÁLISIS FITOQUÍMICO \\ CUALITATIVO DE LOS EXTRACTOS \\ ACUOSOS DE Thalassia testudinum BANKS EX KÖNING ET SIMS DE LA LOCALIDAD DE CHAMPOTÓN, CAMPECHE, MÉXICO, DURANTE EL CICLO ANUAL 2016-2017 \\ QUALITATIVE PHYTOCHEMICAL ANALYSIS OF THE AQUEOUS EXTRACTS OF Thalassia testudinum BANKS EX KÖNING ET SIMS FROM THE AREA OF CHAMPOTON, CAMPECHE, MEXICO, DURING THE ANNUAL CYCLE 2016-2017}

García-Granados, R.U.; F. Cruz-Sosa, F.J. Alarcón-Aguilar, A. Nieto-Trujillo, y M.E. Gallegos-Martínez. ANÁLISIS FITOQUÍMICO CUALITATIVO DE LOS EXTRACTOS ACUOSOS DE Thalassia testudinum BANKS EX KÖNING ET SIMS DE LA LOCALIDAD DE CHAMPOTÓN, CAMPECHE, MÉXICO, DURANTE EL CICLO ANUAL 2016-2017.

QUALITATIVE PHYTOCHEMICAL ANALYSIS OF THE AQUEOUS EXTRACTS OF Thalassia testudinum BANKS EX KÖNING ET SIMS FROM THE AREA OF CHAMPOTON, CAMPECHE, MEXICO, DURING THE ANNUAL CYCLE 2016-2017.

Polibetánica N Núm. 48: 151-168 México. Julio 2019

Instituto Politécnico Nacional $\quad$ DOI: $10.18387 /$ polibotanica.48.12

(c) (i) (-) Este es un artículo de acceso abierto bajo la licencia Creative Commons 4.0 Atribución-No Comercial (CC BY-NC 4.0 Internacional). 


\section{ANÁLISIS FITOQUÍMICO CUALITATIVO DE LOS EXTRACTOS ACUOSOS DE Thalassia testudinum BANKS EX KÖNING ET SIMS DE LA LOCALIDAD DE CHAMPOTÓN, CAMPECHE, MÉXICO, DURANTE EL CICLO ANUAL 2016-2017}

\section{QUALITATIVE PHYTOCHEMICAL ANALYSIS OF THE AQUEOUS EXTRACTS OF Thalassia testudinum BANKS EX KÖNING ET SIMS FROM THE AREA OF CHAMPOTON, CAMPECHE, MEXICO, DURING THE ANNUAL CYCLE 2016-2017}

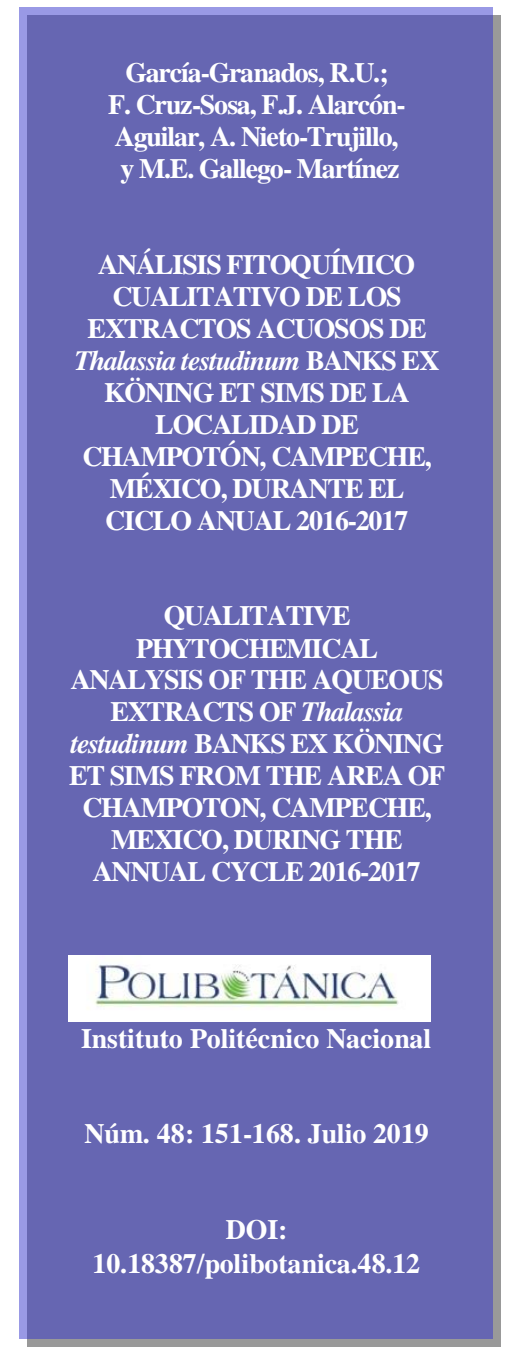

García-Granados, R.U.;

F. Cruz-Sosa, F.J. Alarcón-

Aguilar, A. Nieto-Trujillo,

y M.E. Gallego- Martínez

ANÁLISIS FITOQUÍMICO

CUALITATIVO DE LOS

EXTRACTOS ACUOSOS DE

alassia testudinum BANKS EX

AMPOTÓN, CAMPECHE,

MÉXICO, DURANTE EL

CICLO ANUAL 2016-2017

OUALITATIVE

PHYTOCHEMICAL

NALYSIS OF THE AQUEOUS

EXTRACTS OF Thalassia

CHAMPOTON, CAMPECHE,

MEXICO, DURING THE

ANNUAL CYCLE 2016-2017

\section{POLIBETÁNICA}

Instituto Politécnico Nacional

Núm. 48: 151-168. Julio 2019

10.18387/polibotanica.48.12

\author{
R.U. García-Granados \\ Doctorado en Ciencias Biológicas y de la Salud, UAM-Iztapalapa. \\ Av. San Rafael Atlixco 186, Col. Vicentina, 09340 \\ Ciudad de México, México. \\ F. Cruz-Sosa \\ Departamento de Biotecnología, UAM-Iztapalapa. \\ Av. San Rafael Atlixco 186, Col. Vicentina, 09340 \\ Ciudad de México, México. \\ F.J. Alarcón-Aguilar \\ Departamento de Ciencias de la Salud. UAM-Iztapalapa. \\ Av. San Rafael Atlixco 186, Col. Vicentina, 09340 \\ Ciudad de México, México.
}

A. Nieto-Trujillo
Departamento de Biotecnología, UAM-Iztapalapa.
Av. San Rafael Atlixco 186, Col. Vicentina, 09340
Ciudad de México, México.
M.E. Gallegos-Martínez
Departamento de Hidrobiología, UAM-Iztapalapa.
Av. San Rafael Atlixco 186, Col. Vicentina, 09340
Ciudad de México, México.

RESUMEN: Thalassia testudinum Banks ex Köning et Sims es una fanerógama marina, monocotiledónea, dioica, que se distribuye desde Florida y Golfo de México, hasta las costas de Colombia y Venezuela. Recientes investigaciones han mostrado que T. testudinum presenta actividad antiinflamatoria, antinociceptiva, antioxidante, antiviral, dermorregeneradora, hipoglucemiante, hipolipidémica y neuroprotectora. El objetivo de la presente investigación fue analizar la variabilidad estacional en el contenido de metabolitos de los extractos acuosos de la planta completa, las hojas y el rizoma de $T$. testudinum de tres épocas del año (Nortes, Secas y Lluvias) en la localidad de Champotón Campeche, México. Se determinó la presencia de fenoles, flavonoides, fitoesteroles, cumarinas, diterpenos, triterpenos, saponinas, y azúcares reductores mediante pruebas cualitativas y se cuantificaron las saponinas contenidas en estos extractos, así como su capacidad hemolítica. Los extractos de las hojas de la época de Nortes presentaron la mayor concentración de constituyentes. El contenido de azúcares reductores en los extractos del rizoma estuvo presente durante todo el ciclo anual, mientras que contenido de saponinas fue mayor en los extractos de hojas de la época de Nortes, al igual que su capacidad hemolítica. Los resultados indican que la época de Nortes es la más adecuada para la obtención de material biológico de T. testudinum 
para preparar extractos acuosos con posible aplicación biotecnológica o farmacológica, colectado en la localidad de Champotón, Campeche, México.

Palabras clave: Thalassia testudinum, análisis fitoquímico, pastos marinos, saponinas, variación estacional.

ABSTRACT: Thalassia testudinum Banks ex Köning et Sims is a marine phanerogam, monocotyledonous, dioica that is distributed from Florida and Gulf of Mexico, up to the coasts of Colombia and Venezuela. Recent researches have shown that T. testudinum has antiinflammatory, antinociceptive, antioxidant, antiviral, dermo-regenerative, hypoglycemic, hypolipidemic and neuroprotective activity. The purpose of this investigation was to analyze the seasonal variability in the content of metabolites in the aqueous extracts of the whole plant, the leaves and the rhizome of $T$. testudinum during the seasons of the year (northern, dry and rainy) in Champotón, Campeche, Mexico. The presence of phenols, flavonoids, phytosterols, coumarins, diterpenes, triterpenes, saponins, and reducing sugars was determined by qualitative tests and the saponins contain in these extracts was quantified, as well as its hemolytic capacity. The extracts of the leaves of the northern period presented the highest concentration of constituents. Reducing sugars content in rhizome extracts was present throughout the annual cycle, while the content of saponins was higher in the leave extracts of the northern season, as well as its hemolytic capacity. The results indicate that the northern season is the most suitable for obtaining biological material of $T$. testudinum to prepare aqueous extracts with biotechnological or pharmacological applications, collected in Champoton, Campeche, Mexico.

Key words: Thalassia testudinum, phytochemical analysis, saponins, seagrasses, seasonal variation.

\section{INTRODUCCIÓN}

El pasto marino Thalassia testudinum Banks ex Köning et Sims es una fanerógama marina, monocotiledónea, dioica y es la principal formadora de praderas de pastos marinos en el Caribe, su área de distribución se extiende desde Florida y Golfo de México hasta las costas de Colombia y Venezuela. Se desarrolla en zonas muy someras, hasta unos $14 \mathrm{~m}$ de profundidad, pudiendo quedar expuesta al aire durante mareas bajas extremas (Diaz, Barrios, \& Gómez, 2003).

Diversas investigaciones en los últimos años han demostrado el potencial farmacológico de los extractos de Thalassia testudinum, con efectos analgésicos, antibióticos, antiinflamatorios, antinociceptivos, antioxidantes, antivirales, citoprotectores, dermorregeneradores, hipoglucémicos, hipolipidémicos y neuroprotectores. De los extractos de T. testudinum se han aislado e identificado flavonas glicosiladas, como las talasiolinas A, B y C (De la Torre Núñez, 2014), (Garateix, y otros, 2011), (García-Granados, 2015) (Jensen, y otros, 1998) (Llanio, y otros, 2006) (Menéndez, y otros, 2015) (Regalado, y otros, 2009) (Regalado, y otros, 2012) (Rodeiro, y otros, 2008) (Rowley, y otros, 2002) (Torre-Núñez, y otros, 2012).

El contenido de metabolitos primarios y secundarios, tanto en macrófitas marinas como en fanerógamas terrestres, depende de las condiciones del hábitat, las interacciones ecológicas, el ciclo de vida, la variación estacional, fenómenos meteorológicos y las diferencias genéticas; ante estos múltiples factores considerados como estímulos, hay una biosíntesis, distribución y movilización diferencial de sus metabolitos entre las estructuras de la planta (Arnold \& Schultz, 2002); (Arnold, y otros, 2004); (Gallegos, y otros, 1992); (Kostetsky, y otros, 2004); (Kumar, 1997); (Marbà, y otros, 1994) (Pirc, 1989); (Ramah, y otros, 2014); (Steele, y otros, 2005); (Vergés, y otros, 2007); (Waterman \& Mole, 1994); (Zidorn, 2016). En el presente trabajo se determinó la presencia de fenoles, flavonoides, fitoesteroles, diterpenos, triterpenos, saponinas, y azúcares reductores en extractos acuosos de la planta completa, hojas y rizoma de $T$. testudinum durante el ciclo anual 2016-2017 mediante pruebas cualitativas; además se 
cuantificó el contenido de saponinas y su actividad hemolítica, considerando la variación estacional de la localidad. Esto con la finalidad de conocer las partes de la planta y la temporada con mayor contenido de metabolitos.

\section{MATERIAL Y MÉTODOS}

\section{Área de estudio}

La colecta del material vegetal se realizó en consideración de la variación estacional anual en el estado de Campeche, localidad de Champotón (19 $19^{\circ} 19^{\prime} 50.10^{\prime \prime} \mathrm{N} / 90^{\circ} 43^{\prime} 36.00^{\prime \prime} \mathrm{W}$ ), bajo el permiso de pesca de fomento: DG0PA.00665.060213.0220: época de Nortes $(2016,2017)$, Secas (2017) y Lluvias (2017).

\section{Material biológico y elaboración de extractos}

La colecta del material biológico se hizo manualmente. Los especímenes de T. testudinum se lavaron con agua de mar, se colocaron en bolsas Ziploc con poca agua y sin sedimentos, asignándoles el número de colecta correspondiente a la estación y se preservaron dentro de una hielera a $4{ }^{\circ} \mathrm{C}$. Las muestras colectadas se conservaron a una temperatura de $-20{ }^{\circ} \mathrm{C}$. Se descongelaron y se removieron los organismos epífitos presentes en las hojas y en los rizomas. El pasto marino se deshidrató, pesó y separó en tres secciones: planta completa, hojas y rizoma; cada parte, se molió en un procesador de alimentos. El material vegetal molido (40 g de planta completa, hojas o rizoma) se colocó en un matraz con $500 \mathrm{~mL}$ de agua destilada. La solución se mantuvo en agitación durante $15 \mathrm{~min}$ y se calentó por $10 \mathrm{~min}$ a $80{ }^{\circ} \mathrm{C}$. Posteriormente, cada extracto se incubó a $4{ }^{\circ} \mathrm{C}$ durante 24 h en un matraz cubierto con papel aluminio. Los extractos se filtraron y el filtrado se colocó en un cristalizador dentro de una campana de extracción. El sólido resultante de cada muestra se pesó y conservó a $4{ }^{\circ} \mathrm{C}$ (García-Granados, y otros, 2016).

\section{Análisis fitoquímico cualitativo.}

El análisis fitoquímico cualitativo se realizó siguiendo las metodologías y criterios de evaluación propuestos por (Bulugahapitiya, 2013); (García, y otros, 2003); (Khattak, y otros, 2017); (Yero-Espinosa, y otros, 2017).

En la tabla 1 se muestran los criterios usados para evidenciar la presencia de los constituyentes químicos de T. testudinum. En la tabla 2 se indican los controles positivos utilizados en esta serie de pruebas. Para la detección de alcaloides, lactonas sesquiterpénicas, fitoesteroles, terpenos, glucósidos cardiotónicos, fenoles, taninos, flavonoides, cumarinas, quinonas, azucares reductores y proteínas, se prepararon soluciones de cada uno de los extractos (planta completa, hojas y rizoma) con $500 \mathrm{mg}$ en $10 \mathrm{~mL}$ de agua destilada.

\section{Pruebas para la detección de alcaloides}

- Prueba de Hager: se agregaron 5 gotas de ácido pícrico (J.T. Baker) a las soluciones de los extractos acuosos; la presencia de alcaloides es positiva al precipitar un sólido amarillo (Khattak, y otros, 2017).

- Prueba de Mayer: A $1.0 \mathrm{~mL}$ de las soluciones de cada uno de los extractos acuosos se les añadió dos o tres gotas del reactivo de Mayer. La formación de turbidez o precipitado amarillo indicó la presencia de alcaloides. Cuando la cantidad de alcaloide es menor en el extracto puede sólo generarse turbidez con este reactivo (Bulugahapitiya, 2013).

- Prueba de Wagner: se agregaron 5 gotas de una solución de yoduro de potasio, (reactivo de Wagner) a cada una de las soluciones. Esta prueba de alcaloides es positiva al virar la solución a un color marrón rojizo (Khattak, y otros, 2017).

- Prueba de Dragendorff: se adicionaron 5 gotas del reactivo de Dragendorff a cada una de las soluciones elaboradas con los extractos acuosos, la presencia de alcaloides es positiva al precipitar un sólido rojo (Khattak, y otros, 2017). 
Tabla 1. Criterios utilizados para evidenciar la presencia de constituyentes químicos en el análisis fitoquímico cualitativo.

\begin{tabular}{cc}
\hline Criterio & Nomenclatura \\
\hline Presencia abundante & +++ \\
Presencia notable & ++ \\
Presencia mínima & + \\
Ausencia & - \\
\hline
\end{tabular}

Tabla 2. Controles positivos utilizados en el análisis fitoquímico cualitativo.

\begin{tabular}{ll}
\hline \multicolumn{1}{c}{ Metabolitos } & \multicolumn{1}{c}{ Controles } \\
\hline Alcaloides & Atropina (Sophia) \\
Lactonas sesquiterpénicas & Artemisinina (Sigma) \\
Saponinas & Saponina extracto de Quillaja saponaria Molina (Meyer) \\
Fitoesteroles, & Colesterol (Meyer) \\
Triterpenos & Artemisinina (Sigma) \\
Diterpenos & Artemisinina (Sigma) \\
Glucósidos cardiotónicos & Digoxina (Armstrong) \\
& Ácido gálico (Sigma) \\
Fenoles & Ácido tánico (Indigo) \\
Taninos & Quercetina (Sigma) \\
Flavonoides & Cumarina \\
Cumarinas & Ubiquinona (Telomer) \\
Quinonas & Glucosa (Meyer) \\
Azucares reductores & Albumina (Meyer) \\
Proteínas & Polvo de almendra (Prunus dulcis (L.) Batsch) \\
Lípidos & \\
\hline
\end{tabular}

Pruebas para la detección de lactonas sesquiterpénicas

- Prueba de Baljet: a $5.0 \mathrm{~mL}$ de las soluciones de los extractos acuosos, se adicionó de 3 a 4 gotas del reactivo de Baljet; un cambio de coloración de naranja a rojo demuestra la presencia de lactonas sesquiterpénicas.

\section{Pruebas para la detección de saponinas}

- Prueba de espuma: se diluyeron $500 \mathrm{mg}$ de cada uno de los extractos acuosos de T. testudinum en $10 \mathrm{~mL}$ de agua destilada en un tubo de ensayo. Se agitaron esta serie de soluciones durante $30 \mathrm{~s}$ utilizando el agitador Vortex Genie 2 G-560 y se dejaron reposar $15 \mathrm{~min}$. Una altura de espuma $<5 \mathrm{~mm}$ indica que la prueba es negativa (-), si es de 5-10 $\mathrm{mm}(+)$ el contenido es moderado; una altura $>15 \mathrm{~mm}(+++)$ indica un alto contenido de saponinas (Bulugahapitiya, 2013); (Galindo, y otros, 1989); (García, y otros, 2003).

- Prueba de emulsión: $500 \mathrm{mg}$ de cada uno de los extractos de T. testudinum se disolvieron en $10 \mathrm{~mL}$ de agua destilada. Las soluciones se calentaron en un baño de agua en ebullición y se filtraron. Posteriormente se mezclaron $10 \mathrm{~mL}$ de las soluciones filtradas con $5 \mathrm{~mL}$ de agua destilada, se agitaron durante $30 \mathrm{~s}$ para la formación de espuma. La espuma de cada uno de los extractos se mezcló con tres gotas de aceite de oliva y se volvió a agitar para la formación de una emulsión, una característica de las saponinas (Bulugahapitiya, 2013).

Prueba para la detección de fitoesteroles y triterpenos

- Prueba Libermann-Burchardt: se disolvieron $500 \mathrm{mg}$ de cada uno de los extractos acuosos de T. testudinum en $10 \mathrm{~mL}$ de cloroformo; a una alícuota de $5 \mathrm{~mL}$ se le adicionaron $0.25 \mathrm{~mL}$ de 
anhídrido acético más dos gotas de ácido sulfúrico (J.T. Baker) concentrado. La formación de diferentes colores indica la presencia de fitoesteroles o terpenos. El color verde indica la presencia de fitoesteroles, mientras que de rosa a morado se indica la presencia de terpenos y triterpenos (Bulugahapitiya, 2013).

- Prueba de Salkowski: se disolvieron $500 \mathrm{mg}$ de cada uno de los extractos acuosos de $T$. testudinum en $10 \mathrm{~mL}$ de cloroformo (J.T. Baker), las soluciones se filtraron y se les agregó de dos o tres gotas de ácido sulfúrico concentrado (J.T. Baker); las soluciones se agitaron y se mantuvieron en reposo. La presencia de triterpenos es positiva por el vire de la solución a amarillo, mientras que los fitoesteroles se denotaron por un cambio de coloración a rojo en la capa inferior del tubo de ensayo (Bulugahapitiya, 2013) (Khattak, y otros, 2017).

\section{Prueba para la detección diterpenos}

- Prueba de acetato de cobre: a las soluciones de los extractos acuosos se les agregó de 3 a 4 gotas de una solución de acetato de cobre al 10\% (J.T. Baker). Un cambio de coloración a un color verde esmeralda indica la presencia de diterpenos (Bulugahapitiya, 2013).

\section{Prueba para la detección glucósidos cardiotónicos}

- Prueba Keller-Kilani: se disolvieron $200 \mathrm{mg}$ de los extractos acuosos de T. testudinum en 1 $\mathrm{mL}$ de agua destilada, se agregaron $2 \mathrm{~mL}$ de ácido acético glacial (J.T. Baker) y posteriormente se adicionaron algunas gotas de cloruro férrico al 5\%. Las soluciones se vertieron en tubos de ensayo con $2 \mathrm{~mL}$ de ácido sulfúrico concentrado (J.T. Baker). La formación de un anillo marrón en la interfaz indica la presencia de glucósidos cardiotónicos, al igual que la formación de un anillo violeta debajo del anillo marrón o bien en la fase de ácido acético; un anillo verdoso también puede formarse gradualmente, indicando la presencia de estos compuestos (Bulugahapitiya, 2013).

\section{Prueba para la detección de fenoles}

- Prueba de cloruro férrico al 12.5\% $\left(\mathrm{FeCl}_{3}\right)$ : se agregaron dos a tres gotas de solución de $\mathrm{FeCl}_{3}$ (J.T. Baker) a las soluciones de los extractos acuosos. La transición colorimétrica de verde obscuro a azul índigo indicó la presencia de fenoles, mientras que el color azul completo indica que los taninos de la muestra son hidrolizables. La presencia de taninos condensados en la muestra se confirma por un color verde obscuro (Bulugahapitiya, 2013).

\section{Prueba para la detección de taninos}

- Prueba de precipitación con gelatina: se disolvieron $500 \mathrm{mg}$ de los extractos acuosos de $T$. testudinum en $10 \mathrm{~mL}$ de una solución de gelatina (1\%) y cloruro de sodio $(10 \%)$.

De esta forma, los taninos e incluso los pseudotaninos a altas concentraciones precipitan (Bulugahapitiya, 2013).

\section{Pruebas para la detección de flavonoides}

- Prueba de Shinoda: a $1.0 \mathrm{~mL}$ de los extractos acuosos se le agregó $0.1 \mathrm{~g}$ de limaduras de magnesio y se calentó a $60{ }^{\circ} \mathrm{C}$; posteriormente se adicionaron unas cuantas gotas de ácido clorhídrico (J.T. Baker), por las paredes. Esta prueba es positiva con cambios de coloración a naranja, rojo, rosa, azul o violeta (Bulugahapitiya, 2013).

- Prueba alcalina: las soluciones de los extractos acuosos se trataron con $1.0 \mathrm{~mL}$ de una solución de hidróxido de sodio (J. T Baker), al 40\% dando un fuerte color amarillo. Al añadir $1.0 \mathrm{~mL}$ de ácido clorhídrico (J. T Baker), diluido (10\%) se observa una decoloración, lo que demuestra la presencia de flavonoides (Bulugahapitiya, 2013).

- Prueba de acetato de plomo: Se adicionó $0.5 \mathrm{~mL}$ de una solución de acetato de plomo (Meyer) al $2 \%$ a las soluciones de los extractos acuosos de $T$. testudinum $(500 \mathrm{mg}$ en $10 \mathrm{~mL}$ de agua destilada), un color amarillo indica la presencia de flavonoides (Bulugahapitiya, 2013).

\section{Pruebas para la detección de cumarinas}

- Prueba de fluorescencia: La boca de los tubos de ensayo conteniendo las soluciones de cada uno de los extractos se cubrieron con un círculo de papel de filtro previamente tratado con 
solución de hidróxido de sodio (J. T Baker), 1 N. Se colocó el tubo de ensayo durante unos minutos en agua hirviendo y posteriormente se retiró el papel filtro y se examinó bajo la luz ultravioleta. La aparición de fluorescencia indicó la presencia de cumarinas (Bulugahapitiya, 2013).

\section{Pruebas para la detección de quinonas}

- Prueba de quinonas: se disolvieron $500 \mathrm{mg}$ de cada uno de los extractos de T. testudinum en agua destilada y metanol (Meyer) (1:1) y se le adicionó $1.0 \mathrm{~mL}$ de ácido sulfúrico concentrado (J. T Baker). La formación de color rojo indica la presencia de quinonas.

\section{Pruebas para la detección de azúcares reductores}

- Prueba de Fehling: se agregaron de 2 a 3 gotas del reactivo de Fehling a las soluciones de los extractos acuosos. Un color rojo indica la presencia de azúcares reductores (Khattak, y otros, 2017).

- Prueba de Benedict; De 2 a 3 gotas de la solución de Benedict se agregaron a las soluciones de los extractos acuosos. La presencia de azúcares reductores se confirma por la apariencia de un color rojo ladrillo (Khattak, y otros, 2017).

\section{Prueba para la detección de proteínas}

- Prueba de Biuret: a $100 \mathrm{mg}$ de cada extracto dentro de un tubo de ensayo se agregan 5 a 6 gotas de sulfato cúprico diluido; después se le agregan $5 \mathrm{~mL}$ de una solución de hidróxido de sodio (J.T. Baker) al 40\%. La formación de una coloración violeta o rosa indica la presencia de proteínas dentro del extracto (Bulugahapitiya, 2013) (Khattak, y otros, 2017).

\section{Prueba para la detección de lípidos}

- Prueba de la mancha: se presionó una pequeña cantidad de cada uno de los extractos acuosos entre los pliegues del papel de filtro. En caso de la presencia de lípidos se puede observar una mancha aceitosa translúcida sobre la superficie del papel filtro (Khattak, y otros, 2017).

\section{Cuantificación de saponinas}

Considerando los resultados del análisis fitoquímico cualitativo se procedió a cuantificar el contenido de saponinas por el método de (Khattak, y otros, 2017). Se usaron $10 \mathrm{~mL}$ de agua destilada para disolver $1 \mathrm{~g}$ de los extractos de $T$. testudinum de las plantas completas, hojas y rizomas recolectadas en el ciclo anual 2016-2017; posteriormente cada solución se colocó en un embudo de separación de $250 \mathrm{~mL}$. Cada mezcla fue agitada vigorosamente después de adicionar $10 \mathrm{~mL}$ de éter dimetílico. Después se drenó la capa de éter dimetílico y se agregaron $30 \mathrm{~mL}$ de n-butanol a la fase acuosa, proceso por el cual se precipitaron las saponinas contenidas en cada uno de los extractos. Previamente se pesó el papel filtro (P1) que se utilizó para recoger las saponinas y para desprender las saponinas adheridas en las paredes del embudo de separación, se lavaron en cada proceso de extracción con $10 \mathrm{~mL}$ de solución de $\mathrm{NaCl}$ (Meyer) al 5\%. Para evaporar el disolvente, las muestras obtenidas se colocaron en una campana de extracción de flujo laminar a temperatura ambiente. Ya secos los productos, el papel filtro se pesó nuevamente, obteniéndose los segundos valores (P2).

El contenido de saponinas del extracto se calculó como se indica a continuación:

$$
\begin{gathered}
\text { Cantidad de saponinas }(\mathrm{mg} / \mathrm{g})=\frac{X}{\text { Peso de la muestra }} \\
\% \text { de saponinas }=\frac{X}{\text { Peso de la muestra }} \times 100
\end{gathered}
$$

$\mathrm{X}=$ peso de las saponinas $=\mathrm{P} 2-\mathrm{P} 1$

P1 = Peso del papel filtro.

P2 = Peso del papel filtro + residuo. 


\section{Prueba de hemólisis}

Para observar la actividad hemolítica de los extractos obtenidos de T. testudinum durante este ciclo anual se usó las metodologías modificadas de (Ali \& Al Mutairi, 2015) y (Sotheeswaran, 1988). Se usó un matraz Erlenmeyer de $500 \mathrm{ml}$ para la preparación de los medios. El matraz se llenó con $390 \mathrm{ml}$ de agua destilada y $16 \mathrm{~g}$ de polvo de agar - triptona de soya (Merck). El matraz Erlenmeyer se colocó sobre una parrilla con agitación para llevar la solución a punto de ebullición, lograr su clarificación y su completa disolución; posteriormente se esterilizó en una autoclave a $121{ }^{\circ} \mathrm{C}$ durante 15 minutos. La solución se enfrió a $45^{\circ} \mathrm{C}$ y se adicionaron $28 \mathrm{ml} \mathrm{de}$ sangre al medio disuelto, agitándose suavemente para generar una solución homogénea. Al final se vertieron en cajas de Petri previamente esterilizadas, dejando gelificar a temperatura ambiente durante $20 \mathrm{~min}$.

Par aplicar las muestras se cortaron círculos de papel filtro de $1 \mathrm{~mm}$ de diámetro, los cuales se esterilizaron previamente y se colocaron de forma equidistante sobre el agar-sangre gelificado; a cada uno de los círculos de papel filtro se les agregó $100 \mu \mathrm{l}$ de cada uno de los extractos acuosos de T. testudinum, la saponina o extracto control de Quillaja saponaria (Meyer) que fungió como control positivo y agua estéril como control negativo. La concentración utilizada del control y las muestras problemas fue de $10 \mathrm{mg} / \mathrm{ml}$ y se disolvieron en agua destilada estéril. Cada una de las cajas Petri se mantuvo sellada con papel Parafilm (Bemis) y se incubaron durante $24 \mathrm{~h}$ a temperatura ambiente. En cada placa de agar-sangre se observó un halo o una zona clarificada o de hemolisis alrededor de los círculos de papel filtro, tanto con las muestras problema como en el control positivo, el cual se midió, registrando la distancia desde el punto más alejado de la hemólisis hasta el borde del círculo de papel filtro, utilizando un calibrador vernier digital HER-411 (Steren).

\section{Análisis estadístico}

Todos los experimentos fueron realizados por triplicado y los datos recabados fueron analizados utilizando el paquete estadístico del programa "SPSS Statistics" versión 22 (IBM). Se realizaron análisis de varianza de una vía (ANOVA) y pruebas complementarias de comparación múltiple de medias (Tukey-Kramer), con un nivel de significancia $\mathrm{p} \leq 0.05$.

\section{RESUlTADOs}

Los resultados del análisis fitoquímico cualitativo realizado a cada uno de los extractos acuosos de T. testudinum elaborados con la planta completa, hojas y rizoma, mostraron la presencia de metabolitos secundarios, como fenoles, flavonoides, cumarinas, fitoesteroles, triterpenos, diterpenos y saponinas, en todos los extractos y durante las tres épocas del año de la localidad; siendo mayor su presencia en las épocas de nortes y lluvias para los extractos de hojas; en la época de secas, esta serie de metabolitos secundarios también se detectó en los extractos del rizoma (tabla 3). Los azúcares reductores estuvieron presentes en todos los extractos elaborados con el rizoma, sin importar la variación estacional de la localidad de Champotón, Campeche, México (tabla 3).

Los valores de la prueba de espuma indican que el contenido de saponinas fue mayor en el extracto elaborado con las hojas de T. testudinum de la época de Nortes 2016 y similar al del extracto elaborado con la planta completa de la misma temporada, sin superar al extracto de Quijalla saponaria (Meyer), que fungió como control en esta prueba (tabla 4). Los demás valores de los otros extractos fueron decreciendo en la época de Secas y aumentando paulatinamente en la época de Lluvias y Nortes 2017. Las pruebas estadísticas denotaron diferencias significativas respecto al extracto elaborado con las hojas de la época de Nortes 2016 (tabla 4). 
Tabla 3. Variación estacional en el contenido de metabolitos de los extractos acuosos de la planta completa, las hojas y el rizoma T. testudinum, durante el ciclo anual 2016-2017.

\begin{tabular}{|c|c|c|c|c|}
\hline Constituyentes & Época/Prueba & $\begin{array}{c}\text { Planta } \\
\text { completa }\end{array}$ & Hojas & Rizoma \\
\hline \multirow[t]{8}{*}{ Fenoles } & Nortes 2016 & & & \\
\hline & Cloruro férrico & + & +++ & + \\
\hline & Época de Secas 2017 & & & \\
\hline & Cloruro férrico & ++ & + & +++ \\
\hline & Lluvias 2017 & & & \\
\hline & Cloruro férrico & + & ++ & + \\
\hline & Nortes 2017 & & & \\
\hline & Cloruro férrico & ++ & +++ & + \\
\hline \multirow[t]{16}{*}{ Flavonoides } & Nortes 2016 & & & \\
\hline & Shinoda & ++ & +++ & + \\
\hline & Reacción alcalina & ++ & ++ & + \\
\hline & Acetato de plomo & ++ & +++ & + \\
\hline & Secas 2016 & & & \\
\hline & Shinoda & ++ & + & +++ \\
\hline & Reacción alcalina & ++ & + & ++ \\
\hline & Acetato de plomo & ++ & + & +++ \\
\hline & Lluvias 2017 & & & \\
\hline & Shinoda & + & +++ & + \\
\hline & Reacción alcalina & ++ & ++ & + \\
\hline & Acetato de plomo & + & +++ & + \\
\hline & Nortes 2017 & & & \\
\hline & Shinoda & ++ & +++ & + \\
\hline & Reacción alcalina & ++ & ++ & + \\
\hline & Acetato de plomo & ++ & +++ & + \\
\hline \multirow[t]{9}{*}{ Cumarinas } & Nortes 2016 & & & \\
\hline & Fluorescencia & ++ & +++ & + \\
\hline & Secas 2017 & & & \\
\hline & Fluorescencia & ++ & + & +++ \\
\hline & Lluvias 2017 & & & \\
\hline & Fluorescencia & + & +++ & + \\
\hline & Nortes 2017 & & & \\
\hline & Fluorescencia & ++ & +++ & + \\
\hline & Nortes 2016 & & & \\
\hline \multirow{7}{*}{ Saponinas } & Emulsión & ++ & +++ & ++ \\
\hline & Secas 2017 & & & \\
\hline & Emulsión & ++ & + & ++ \\
\hline & Lluvias 2017 & & & \\
\hline & Emulsión & + & ++ & + \\
\hline & Nortes 2017 & & & \\
\hline & Emulsión & ++ & ++ & ++ \\
\hline \multirow[t]{6}{*}{ Fitoesteroles } & Nortes 2016 & & & \\
\hline & Lieber-Buchart & +++ & +++ & +++ \\
\hline & Salkowski & ++ & +++ & ++ \\
\hline & Secas 2017 & & & \\
\hline & Lieber-Buchart & ++ & + & +++ \\
\hline & Salkowski & ++ & + & +++ \\
\hline
\end{tabular}




\begin{tabular}{|c|c|c|c|c|}
\hline Constituyentes & Época/Prueba & $\begin{array}{c}\text { Planta } \\
\text { completa }\end{array}$ & Hojas & Rizoma \\
\hline & Lluvias 2017 & & & \\
\hline & Lieber-Buchart & + & ++ & + \\
\hline & Salkowski & ++ & ++ & + \\
\hline & Nortes 2017 & & & \\
\hline & Lieber-Buchart & ++ & +++ & ++ \\
\hline & Salkowski & ++ & +++ & + \\
\hline \multirow[t]{8}{*}{ Triterpenos } & Nortes 2016 & & & \\
\hline & Salkowski & + & +++ & + \\
\hline & Secas 2017 & & & \\
\hline & Salkowski & ++ & ++ & +++ \\
\hline & Lluvias 2017 & & & \\
\hline & Salkowski & + & + & ++ \\
\hline & Nortes 2017 & & & \\
\hline & Salkowski & + & +++ & ++ \\
\hline \multirow[t]{8}{*}{ Diterpenos } & Nortes 2016 & & & \\
\hline & Acetato de cobre & ++ & +++ & ++ \\
\hline & Secas 2017 & & & \\
\hline & Acetato de cobre & ++ & ++ & +++ \\
\hline & Lluvias 2017 & & & \\
\hline & Acetato de cobre & + & ++ & + \\
\hline & Nortes 2017 & & & \\
\hline & Acetato de cobre & ++ & +++ & ++ \\
\hline \multirow{12}{*}{$\begin{array}{c}\text { Azúcares } \\
\text { reductores }\end{array}$} & Nortes 2016 & & & \\
\hline & Fehling & ++ & + & +++ \\
\hline & Benedict & ++ & + & +++ \\
\hline & Secas 2017 & & & \\
\hline & Fehling & ++ & + & +++ \\
\hline & Benedict & ++ & + & +++ \\
\hline & Lluvias 2017 & & & \\
\hline & Fehling & ++ & + & +++ \\
\hline & Benedict & ++ & + & +++ \\
\hline & Nortes 2017 & & & \\
\hline & Fehling & ++ & + & +++ \\
\hline & Benedict & ++ & + & +++ \\
\hline
\end{tabular}


Tabla 4. Prueba de espuma de los extractos acuosos de la planta completa, las hojas y el rizoma T. testudinum durante el ciclo anual 2016-2017.

\begin{tabular}{|c|c|c|c|}
\hline Época del año & Extractos acuosos & Espuma (cm) & $\mathbf{C V}$ \\
\hline \multirow{3}{*}{ Nortes 2016} & Planta completa & $3.3 \pm .052 *$ & 1.7 \\
\hline & Hojas & $3.7 \pm .152$ & 4.0 \\
\hline & Rizoma & $3.1 \pm .057 * \#$ & 1.8 \\
\hline \multirow{3}{*}{ Secas 2017} & Planta completa & $2.0 \pm .100^{* \#}$ & 5.0 \\
\hline & Hojas & $1.8 \pm .251 * \#$ & 13.4 \\
\hline & Rizoma & $2.1 \pm .152^{* \#}$ & 7.0 \\
\hline \multirow{3}{*}{ Lluvias 2017} & Planta completa & $2.2 \pm .200^{* \#}$ & 9.0 \\
\hline & Hojas & $2.9 \pm .152 * \#$ & 5.2 \\
\hline & Rizoma & $2.4 \pm .173$ *\# & 7.2 \\
\hline \multirow{3}{*}{ Nortes 2017} & Planta completa & $3.0 \pm .264^{* \#}$ & 8.8 \\
\hline & Hojas & $3.1 \pm .100^{* \#}$ & 3.2 \\
\hline & Rizoma & $2.9 \pm .200^{* \#}$ & 6.8 \\
\hline Control & Saponina (Quillaja saponaria) (Meyer) & $4.1 \pm .100$ & 2.4 \\
\hline
\end{tabular}

Los datos representan la media \pm desviación estándar $(\mathrm{n}=3)$. *Diferencia significativa respecto al control. \#Diferencia significativa respecto al extracto elaborado con las hojas de T. testudinum de la época de Nortes 2016 (Tukey-Kramer, $\mathrm{P}<0.05)$. CV = coeficiente de variación.

En relación con el contenido de saponinas de los extractos se observó que el contenido del extracto de Quillaja saponaria (Meyer) está por encima de todos los extractos elaborados con T. testudinum (tabla 5); sin embargo, el contenido de saponinas de ambos extractos elaborados con las hojas de las épocas de Nortes 2016 y 2017 fue similar y mayor a los demás extractos analizados por medio de esta metodología. Las pruebas estadísticas aplicadas a esta serie de datos muestran diferencias significativas respecto al extracto elaborado con las hojas de la época de Nortes y al extracto control (tabla 5).

Tabla 5. Cuantificación de saponinas de los extractos acuosos de la planta completa, las hojas y el rizoma $T$. testudinum, durante el ciclo anual 2016-2017.

\begin{tabular}{|c|c|c|c|c|}
\hline Época del año & Extractos acuosos & $\begin{array}{c}\text { Saponinas } \\
\text { mg/g extracto }\end{array}$ & $\%$ & CV \\
\hline \multirow{3}{*}{ Nortes 2016} & Planta completa & $277.0 \pm 3.412 * \#$ & 27.7 & 1.2 \\
\hline & Hojas & $314.7 \pm 1.474^{*}$ & 31.4 & .5 \\
\hline & Rizoma & $261.3 \pm 2.715$ *\# & 26.1 & 1.0 \\
\hline \multirow{3}{*}{ Secas 2017} & Planta completa & $169.1 \pm 0.642 * \#$ & 16.9 & .4 \\
\hline & Hojas & $153,8 \pm 2.433^{* \#}$ & 15.3 & 1.5 \\
\hline & Rizoma & $170.2 \pm 1.058 * \#$ & 17.0 & .6 \\
\hline \multirow{3}{*}{ Lluvias 2017} & Planta completa & $187.6 \pm 0.692 * \#$ & 18.7 & .4 \\
\hline & Hojas & $244.8 \pm 1.113^{* \#}$ & 24.4 & .5 \\
\hline & Rizoma & $204.2 \pm 1.928^{*}$ & 20.4 & .9 \\
\hline \multirow{3}{*}{ Nortes 2017} & Planta completa & $255.0 \pm 0.971 * \#$ & 25.5 & .4 \\
\hline & Hojas & $312.8 \pm 0.916^{*}$ & 31.2 & .3 \\
\hline & Rizoma & $246.2 \pm 0.529 * \#$ & 24.6 & .2 \\
\hline Control & $\begin{array}{l}\text { Saponina (Quillaja } \\
\text { saponaria) (Meyer) }\end{array}$ & $342.3 \pm 3.910$ & 34.2 & 1.1 \\
\hline
\end{tabular}

Los datos expuestos representan la media \pm desviación estándar $(\mathrm{n}=3)$. *Diferencia significativa respecto al control. \#Diferencia significativa respecto al extracto elaborado con las hojas de T. testudinum de la época de Nortes 2016 (Tukey-Kramer, $\mathrm{P}<0.05)$. CV = coeficiente de variación. 
El diámetro de los halos generados alrededor los tratamientos aplicados sobre el agar-sangre fueron mayores con los extractos elaborados con la planta completa y las hojas de la época de Nortes 2016 y de la misma manera con el extracto de las hojas de la época de Nortes 2017 , debido a esto, las pruebas estadísticas encontraron diferencias significativas en relación con los demás extractos y todos los extractos en general en comparación al extracto control de Quillaja saponaria (Meyer) (tabla 6).

Tabla 6. Hemólisis observada con los extractos acuosos de la planta completa, las hojas y el rizoma T. testudinum, durante el ciclo anual 2016-2017.

\begin{tabular}{cccc}
\hline Época del año & Extractos acuosos & Hemolisis $(\mathbf{m m})$ & CV \\
\hline \multirow{3}{*}{ Nortes 2016} & Planta completa & $3.1 \pm .153^{*}$ & 4.8 \\
& Hojas & $3.4 \pm .058^{*}$ & 1.6 \\
& Rizoma & $2.9 \pm .100^{* \#}$ & 3.4 \\
\hline \multirow{3}{*}{ Secas 2017} & Planta completa & $1.7 \pm .115^{* \#}$ & 6.6 \\
& Hojas & $1.6 \pm .058^{* \#}$ & 3.6 \\
& Rizoma & $1.8 \pm .058^{* \#}$ & 3.1 \\
\hline \multirow{3}{*}{ Lluvias 2017} & Planta completa & $2.0 \pm .058^{* \#}$ & 2.8 \\
& Hojas & $2.7 \pm .058^{* \#}$ & 2.1 \\
& Rizoma & $2.4 \pm .208^{* \#}$ & 8.7 \\
\hline \multirow{2}{*}{ Nortes 2017} & Planta completa & $2.8 \pm .058^{* \#}$ & 2.0 \\
& Hojas & $3.3 \pm .115^{*}$ & 3.4 \\
Control & Rizoma & $2.7 \pm .200^{* \#}$ & 7.4 \\
\hline & Saponina (Quillaja saponaria) (Meyer) & $3.7 \pm .138$ & 3.7
\end{tabular}

Los datos expuestos representan la media \pm desviación estándar $(\mathrm{n}=3)$. *Diferencia significativa respecto al control. \#Diferencia significativa respecto al extracto elaborado con las hojas de T. testudinum de la época de Nortes 2016 (Tukey-Kramer, $\mathrm{P}<0.05)$. CV = coeficiente de variación.

\section{DISCUSIÓN}

Los resultados del análisis fitoquímico cualitativo muestran que los extractos de este ciclo anual presentan una importante composición fenólica, representada por fenoles, flavonoides y cumarinas. Las fanerógamas marinas en general son una fuente rica de compuestos fenólicos, como ácidos fenólicos, ácidos fenólicos sulfatados, cumarinas, flavonas, taninos y lignanos, cuya biosíntesis es por la vía del ácido shikímico y son el reflejo de interacciones bióticas y abióticas (Arnold \& Schultz, 2002); (Arnold, y otros, 2004); (Arnold, y otros, 2008); (Mc Millan, y otros, 1980); (Papenbrock, 2012); (Sieg \& Kubanek, 2013); (Vergeer \& Develi, 1997).

En este análisis, los extractos de hojas de las épocas de Nortes y Lluvias mostraron la mayor presencia de compuestos fenólicos, mientras que en la época de Secas en los extractos elaborados con el rizoma. Esto coincide con lo reportado por (García-González, y otros, 2011); (Hernández, y otros, 2016) y (Rowley, y otros, 2002), quienes documentaron la estrecha relación entre variación estacional, localidad y biosíntesis de compuestos fenólicos en $T$. testudinum.

Varios reportes indican que la biosíntesis de compuestos fenólicos es esencial en los pastos marinos para inhibir el crecimiento de microorganismos que pudieran afectar su viabilidad, la tolerancia ante condiciones adversas, la competencia por el espacio con otras macrófitas marinas y evitar la herbívora (Arnold, y otros, 2008) (Dumay, y otros, 2004); (Hernández, y otros, 2016); (Jensen, y otros, 1998); (Mc Millan, 1984); (Trevathan-Tackett, y otros, 2015); 
(Vergeer, y otros, 1995); (Vergeer \& Develi, 1997). A su vez, en todos los extractos acuosos predominó la presencia de metabolitos secundarios derivados del ácido mevalónico (fitoesteroles, triterpenos, diterpenos y saponinas), siendo las saponinas las más abundantes.

En especies de fanerógamas marinas, como Cymodocea nodosa (Ucria) Ascherson, $C$. rotundata Ascherson \& Schweinfurth, C. serrulata (R.Brown) Ascherson \& Magnus, Halophila pinnifolia (Miki) den Hartog, H. ovalis (R. Brown) J.D. Hooker, Posidonia oceanica (Linneaus) Delile, Syringodium isoetifolium (Ascherson) Dandy y Thalassia hemprichii (Ehrenberg) Ascherson, se han encontrado también saponinas, fitoesteroles y terpenos; de manera homóloga como en las fanerógamas terrestres, también fungen como fitoalexinas. (Ali, y otros, 2012); (Berfad \& Alnour, 2014); (Kontiza, y otros, 2008); (Hammami, y otros, 2013); (Mani, y otros, 2012); (Ragupathi Raja Kannan, y otros, 2013); (Sangeetha \& Asokan, 2016); (Schmelz, y otros, 2014).

Las saponinas estuvieron presentes en todos los extractos acuosos elaborados durante este ciclo anual, incluyendo los valores más bajos que se presentaron en el extracto elaborado con las hojas en la época de secas. En este caso, la media de la altura de la columna de espuma fue de $1.8 \mathrm{~cm}$ y, de acuerdo con la escala de Harborne (1989) para la detección de saponinas, la prueba es positiva con valores de altura de la columna de espuma menor o igual a $2 \mathrm{~cm}$.

Los valores más altos de la columna de espuma se encontraron con los extractos elaborados con la planta completa y las hojas de la época de Nortes. En este sentido, los resultados obtenidos con todos los extractos, incluyendo el extracto control, exceden a la máxima cantidad detectable considerando los criterios expuestos por (Galindo, y otros, 1989) y (García, y otros, 2003). Conforme a la prueba de emulsión se puede decir en general que la mayoría de los extractos pueden ser útiles como emulsificantes.

En relación con la cuantificación de saponinas, se observó de manera homóloga el mismo comportamiento que con la altura de la columna de espuma: el mayor contenido se encontró en los extractos elaborados con las hojas de la época de Nortes, pero su contenido no fue mayor que el extracto de Quillaja saponaria (Meyer), que fungió como control.

Loa mayores diámetros que presentaron hemolisis en las placas de agar-sangre se encontraron en los extractos de la época de Nortes 2016 elaborados con las hojas y la planta completa, al igual que el extracto elaborado con las hojas en la época de Nortes 2017. De esta forma, los resultados obtenidos en la prueba de espuma corresponden bien con la cuantificación de saponinas.

En general las saponinas son consideradas como agentes hemolíticos en pruebas in vitro, esto se debe a su interacción con los esteroles de la membrana eritrocitaria, ocasionando un daño irreversible a la bicapa lipídica. Esta acción genera un aumento de la permeabilidad de la membrana, generando un cambio en el gradiente iónico. En este caso el sodio y el agua entran, mientras que el potasio sale, lo cual genera la total disrupción de la membrana, permitiendo de este modo la salida de la hemoglobina (Bauman, y otros, 2000); (Bruneton, 2001); (Gauthier, y otros, 2009); (Melzig, y otros, 2005).

La hemólisis que pudiese generar una saponina es totalmente dependiente de su composición estructural (Bauman, y otros, 2000); (Bruneton, 2001); (Chwalek, y otros, 2006); (Hernández R., 1997). Tomando en consideración lo anterior, como perspectivas es importante elucidar la o las estructuras de las saponinas contenidas en estos extractos acuosos de esta fanerógama marina y aunque $T$. testudinum no es una planta de uso popular, queda demostrado en este estudio la actividad hemolítica de sus saponinas, por lo que es importante realizar estudios toxicológicos, ya que si las saponinas encontradas tienen características estructurales similares a las contenidas en el extracto de Quillaja saponaria (control) y de igual forma son inocuas a 
bajas concentraciones, podrían ser útiles en la industria alimenticia, cosmética, farmacológica, fotográfica y minera (Cheeke, 2000).

Las saponinas, poseen una amplia actividad biológica y farmacológica, destacándose su efecto antiagregante plaquetario, antibacterial, antifúngica, antiinflamatoria, antioxidante, antiprotozoaria, antiviral, broncolítica, citotóxica, espasmódica, hipolipemiante, hipoglucemiante, inmunoestimulante, insecticida, neuroprotectora y piscida, pero en un sentido más ecológico el contenido de saponinas nos habla indirectamente de la hostilidad del ambiente en el que se desarrolla, ya que conforman parte del sistema de defensa de una planta, entrando también en la categoría de fitoanticipinas o fitoprotectores (Francis, y otros, 2002); (MenaValdés, y otros, 2015); (Morrissey \& Osbourn, 1999). De acuerdo con estas características el potencial de los extractos de T. testudinum provenientes de localidad Champotón, podría ser diverso en la industria farmacológica y biotecnológica.

La presencia de estos metabolitos secundarios, al igual que los compuestos fenólicos, fue mayor en las hojas en las épocas de Nortes y Lluvias, donde posiblemente la precipitación y los fenómenos meteorológicos, así como los cambios en la dinámica de la columna de agua, como la resuspensión del sedimento, aunado al constante aporte de nutrientes del río Champotón y sus descargas municipales, sean los factores principales en la inducción de la biosíntesis de estos metabolitos secundarios, ya que el estrés lumínico generado por la turbidez de la columna de agua genera una mayor densidad de hojas, cuyo efecto es la utilización de la luz para una mayor actividad metabólica; por lo tanto, es de esperar que ante estas condiciones los metabolitos detectados en los análisis fitoquímicos cualitativos estén presentes en las hojas (López-Sánchez, 2012).

La detección de azucares reductores en todos los extractos elaborados con el rizoma, sin importar la variación estacional, demuestra el papel de esta estructura en el almacenamiento de energía, ya que se acumulan durante períodos en donde la producción excede la respiración o cuando la producción es baja. Por otra parte, es importante resaltar que los niveles de carbohidratos en el rizoma son un buen indicador de la salud de las fanerógamas marinas (Carlson Jr., y otros, 1994); (Dawes \& Lawrence, 1980); (Lee \& Dunton 1996).

Considerando la información anterior es de suma importancia tomar en cuenta que la producción y acumulación de metabolitos secundarios también es un indicador del estrés fisiológico inducido por factores antropogénicos. De hecho, los resultados de análisis fitoquímicos cualitativos de forma indirecta pueden reflejar el estado de salud de una pradera de pastos marinos.

Yero-Espinosa et al. (2017) publicaron una investigación realizada con pruebas fitoquímicas cualitativas, en la cual se corroboró que la producción de metabolitos secundarios es multifactorial. Este grupo de trabajo documentó la presencia de alcaloides, fenoles, flavonoides, taninos, cumarinas y azúcares reductores, en sus extractos acuoso y etanólico elaborados con la planta completa de T. testudinum y cuyo material biológico fue colectado en el Caribe. En el presente estudio se observó la presencia de fenoles, flavonoides, cumarinas, fitoesteroles, triterpenos, diterpenos, saponinas y azúcares reductores, coincidiendo en algunos metabolitos el análisis del grupo de trabajo anterior y con los análisis que Regalado et al. (2012) realizaron con un extracto hidroalcohólico de T. testudinum colectada en el Caribe.

Al igual que Regalado et al. (2012) y Yero-Espinosa et al. (2017) no se reportó la presencia de glucósidos cardiotónicos o quinonas, pero existen reportes en la literatura que especies como Cymodocea rotundata, Halophila pinnifolia, H. ovalis y Syringodium isoetifolium contienen este tipo de metabolitos. Por su parte, las quinonas se han encontrado en especies como Thalassia hemprichii, Halophila beccarii Ascherson, Halodule pinifolia, Cymodocea rotundata y C. serrulata (Girija, y otros, 2013); (Ragupathi Raja Kannan, y otros, 2013); (Vani, Murthy, \& Devi, 2019). 
Por lo tanto, el contenido de metabolitos en los extractos acuosos o etanólicos de T. testudinum depende del sitio de la colecta y de la época del año. Diversos estudios fitoquímicos alrededor del mundo muestran que los pastos marinos tienen metabolitos con potencial aplicación farmacológica y biotecnológica, donde la fenología ahora debe ser considerada como una herramienta principal en la búsqueda de compuestos bioactivos.

\section{CONCLUSIÓN}

Los resultados muestran que la época de Nortes es la más adecuada para la obtención de extractos acuosos y moléculas con posible potencial en diversas industrias a partir de material biológico de $T$. testudinum colectado en la localidad de Champotón, Campeche, México. Son necesarios estudios fenológicos complementarios para dar continuidad a estas investigaciones, así como lograr identificar, caracterizar y cuantificar a las moléculas contenidas en estos productos, con la posibilidad de ahondar en el conocimiento de las posibles variantes ambientales responsables de su biosíntesis.

\section{AgradecimiEnTos}

Se agradece al programa de Doctorado en Ciencias Biológicas y de la Salud - División de Ciencias Biológicas y de la Salud, UAM-Iztapalapa que pertenece al Programa Nacional de Posgrados de Excelencia del CONACyT.

\section{LITERATURA CITADA}

Ali, M. S., Ravikumar, S., \& Beula, J. M. (2012). Bioactivity of seagrass against the dengue fever mosquito Aedes aegypti larvae. Asian Pacific Journal of Tropical Biomedicine, 2(7), 570-573. doi:10.1016/S2221-1691(12)60099-9

Arnold, T. M., Tanner, C. E., Rothen, M., \& Bullington , J. (2008). Wound-induced accumulations of condensed tannins in turtlegrass, Thalassia testudinum. Aquatic Botany, 89(1), 27-33. doi:10.1016/j.aquabot.2008.02.001

Arnold, T., \& Schultz, J. (2002). Induced sink strength as a prerequisite for induced tannin biosynthesis in developing leaves of Populus. Oecologia New Phytologist, 130, 585593. doi:10.1007/s00442-001-0839-7

Arnold, T., Appel, H., Patel, V., Stocum, E., Kavalier, A., \& Schultz, J. (2004). Carbohydrate translocation determines the phenolic content of Populus foliage: A test of the sinksource model of plant defense. New Phytologist, 164(1), 157-164. doi:10.1111/j.14698137.2004.01157.x

Berfad, M. A., \& Alnour, T. (2014). Phytochemical analysis and antibacterial activity of the 5 different extract from the seagrasses Posidonia oceanica. Journal of Medicinal Plants Studies, 15(24), 15-18.

Bulugahapitiya, V. P. (2013). Plants based natural products extraction, isolation and phytochemical screening methods (1ra. ed.). Matara, Sri Lanka: Indika Graphics.

De la Torre Núñez, E. P. (2014). Evaluación teratogénica y embriotóxica del extracto hidroalcóholico de la planta marina Thalassia testudium. Morfovirtual. 2do Congreso Virtual de Ciencias Morfológicas. Segunda Jornada Científica Virtual de la Cátedra Santiago Ramón y Cajal, 1-13.

Diaz, J. M., Barrios, L. M., \& Gómez, D. I. (2003). Fishing for life (also known as improving local managemet of tropical costal resources in the face of climate change for economic wellbeing of local and vulnerable communities) View project. Monitoreo de la biodiversidad y los ecosistemas marinos y costeros. doi:10.13140/2.1.3025.0566 
Dumay, O., Costa, J., Desjobert, J. M., \& Pergent, G. (2004). Variations in the concentration of phenolic compounds in the seagrass Posidonia oceanica under conditions of competition. Phytochemistry, 3211-3220. doi:10.1016/j.phytochem.2004.09.003

Galindo, W., Rosales, M., Murgueitio, E., \& Larrahondo, J. (1989). Sustancias antinutricionalesen las hojas de árboles forrajeros. Livestock Research for Rural Development, 1, 1-6.

Gallegos, M. E., Merino, M., Marbá, N., \& Duarte, C. M. (1992). Flowering of Thalassia testudinum banks ex König in the Mexican Caribbean: age-dependence and interannual variability. Aquatic Botany, 43(3), 249-255. doi:10.1016/0304-3770(92)90070-Y

Garateix, A., Salceda, E., Menéndez, R., Regalado, E. L., López, O., García, T., . . . Soto, E. (2011). Antinociception produced by Thalassia testudinum extract BM-21 is mediated by the inhibition of acid sensing ionic channels by the phenolic compound thalassiolin B. Molecular Pain, 7(1), 10. Obtenido de https://doi.org/10.1186/1744-8069-7-10

García, D. E., Ojeda, F., \& Montejo , I. (2003). Evaluación de los principales factores que influyen en la composición fitoquímica de Morus alba (Linn.). Análisis cualitativo de metabolitos secundarios. Pastos y Forrajes, 26(4), 335-346.

García-González, K. L., Iglesias-Valdez, O., Laguna, A., Díaz-Martínez, M., \& GonzálezLavaut, J. A. (2011). Antioxidant effect and polyphenol content of Syringodium filiforme (Cymodoceaceae). Revista de Biología Tropical, 59(1), 465-472.

García-Granados, R. U. (2015). Efecto hipoglucémico, hipolipidémico y citotóxico de macroalgas y pastos marinos del Golfo de México. Tesis de Maestría en Ciencias (Biología).UAM-Iztapalapa, 96 p.

García-Granados, R. U., Alarcón-Aguilar, F., Gallegos-Martínez, M. E., \& De Lara-Isassi, G. (2016). Tratamiento subcrónico en ratones diabéticos con Caulerpa sertularioides (Chlorophyta) y Spyridia filamentosa (Rhodophyta). Hidrobiológica, 26(2), 269-276. doi:10.24275/uam/izt/dcbs/hidro/2016v26n2/DeLara

Hammami, S., Salem, A. B., Ashour, M. L., Cheriaa, J., Graziano, G., \& Mighri, Z. (2013). A novel methylated sesquiterpene from seagrass Posidonia oceanica (L.) Delile. Natural Product Research, 27(14), 1265-1270. doi:10.1080/14786419.2012.725401

Hernández, Y., González, K., Valdés-Iglesias, O., Zarabozo, A., Portal, Y., Laguna, A., \& Gutiérrez, R. (2016). Seasonal behavior of Thalassia testudinum (Hydrocharitaceae) metabolites. Revista de Biología Tropical, 64, 1527-1535. doi:10.15517/rbt.v64i4.21037

Jensen, P. R., Jenkins, K. M., Porter, D., \& Fenical, W. (1998). Evidence that a new antibiotic flavone glycoside chemically defends the sea grass Thalassia testudinum against zoosporic fungi. Applied and Environmental Microbiology, 64(4), 1490-1496. doi:10.1021/jacs.7b12897

Khattak, U., Ullah, R., Khan, S. A., Barkatullah, Ullah, S., \& Saima. (2017). Pharmacognostic evaluation and analgesic efficacy of ethanolic extract of Euphorbia dracunculoides L. . Pharmacognosy Journal, 9(5), 644-653. doi:10.5530/pj.2017.5.102

Kontiza, I., Stavri, M., Zloh, M., Vagias, C., Gibbons, S., \& Roussis, V. (2008). New metabolites with antibacterial activity from the marine angiosperm Cymodocea nodosa. Tetrahedron, 64(8), 1696-1702. doi:10.1016/j.tet.2007.12.007

Kostetsky, E. Y., Goncharova, S. N., Sanina, N. M., \& Shnyrov, V. L. (2004). Season influence on lipid composition of marine macrophytes. Botanica Marina, 47(2), 134-139. doi:10.1515/BOT.2004.013

Kumar, R. (1997). Anti- nutritional factors. The potential risks of toxicity and the methods to alleviate them. En S. A. PUGLIESE, \& S. A. PUGLIESE (Ed.), Legumes trees and other fodder trees as protein sourcefor livestock. Food and Agriculture Organization of the United Nations (FAO). Roma.

Llanio, M., Fernández, M. D., Cabrera, B., Bermejo, P., Abad, M., Payá, M., \& Alcaraz, M. J. (2006). The marine plant Thalassia testudinum anti-inflammatory and analgesic properties. Pharmacology On line, 3, 594-600. 
Mani, A. E., Bharathi, V., \& Patterson, J. (2012). Antibacterial activity and preliminary phytochemical analysis of sea grass Cymodocea rotundata. International Journal of Microbiological Research, 3, 99-103.

Marbà, N., Gallegos, M. E., Merino, M., \& Duarte, C. M. (1994). Vertical growth of Thalassia testudinum: seasonal and interannual variability. Aquatic Botany, 47(1), 1-11. doi:10.1016/0304-3770(94)90043-4

Mc Millan, C. (1984). The condensed tannins (proanthocyanidins) in seagrasses. Aquatic Botany, 20, 351-357. doi:10.1016/0304-3770(84)90099-8

Mc Millan, C., Zapata, O., \& Escobar, L. (1980). Sulphated phenolic compounds in seagrasses. Aquatic Botany, 8, 267-278. doi:10.1016/0304-3770(90)90055-8

Menéndez, R., García, T., Marrero, G., Morales, R. A., Garateix, A., Regalado, E., \& Valdes, O. (2015). Neuroprotective effects of Thalassia testudinum leave extract BM-21 on global ischemia in mongolian gerbils. American Journal of Biomedical Sciencies, 7(4), 229-241. doi:10.5099/aj150400229

Papenbrock, J. (2012). Highlights in seagrasses, phylogeny, physiology, and metabolism: What makes them special? . ISRN Botany, 1-15. doi:10.5402/2012/103892

Pirc, H. (1989). Seasonal changes in soluble carbohydrates, starch, and energy content in mediterranean seagrasses. Marine Ecology, 10(2), 97-105. doi:10.1111/j.14390485.1989.tb00068.x

Ragupathi Raja Kannan, R., Arumugam, R., Iyapparaj, P., Thangaradjou, T., \& Anantharaman, P. (2013). In vitro antibacterial, cytotoxicity and haemolytic activities and phytochemical analysis of seagrasses from the Gulf of Mannar, South India. Food Chemistry, 136(3-4), 1484-1489. doi:10.1016/j.foodchem.2012.09.006

Ramah, S., Etwarysing, L., Auckloo, N., Gopeechund, A., Bhagooli, R., \& Bahorun, T. (2014). Prophylactic antioxidants and phenolics of seagrass and seaweed species: A seasonal variation study in a Southern Indian Ocean Island, Mauritius.,. Internet Journal of Medical Update, 9(1), 27-37.

Regalado, E. L., Menéndez, R., Valdés, O., Morales, R. A., Laguna, A., Thomas, O. P., . . . Kijjoa, A. (2012). Phytochemical analysis and antioxidant capacity of BM-21, a bioactive extract rich in polyphenolic metabolites from the Sea Grass Thalassia testudinum. Natural Product Communications, 7, 47-50.

Regalado, E. L., Rodríguez, M., \& Menéndez, R. (2009). Repair of UVB-damaged skin by the antioxidant sulphated flavone glycoside thalassiolin B isolated from the marine plant Thalassia testudinum Banks ex König. Marine Biotechnology, 11(1), 74-80. doi:10.1007/s10126-008-9123-8

Rodeiro, I., Donato, M. T., Martínez, I., Hernández, I., Garrido, G., González-Gavaut, J., . . . Gómez-Lechón, M. J. (2008). Potential hepatoprotective effects of new cuban natural products in rat hepatocytes culture. Toxicology in Vitro, 22(5), 1242-1249.

Rowley, D. C., Hansen, M., Rhodes, D., Sotriffer, C. A., Ni, H., McCammon, J. A., \& Fenical, W. (2002). Thalassiolins A-C: New marine-derived inhibitors of HIV cDNA integrase. Bioorganic and Medicinal Chemistry, 10(11), 3619-3625. doi:10.1016/S09680896(02)00241-9

Sangeetha, J., \& Asokan, S. (2016). Phytochemical analysis and antibacterial activity of the three different seagrass extracts . International Journal of Advanced Research, 4, 1451-1457. doi:10.21474/IJAR01/471

Schmelz, E., Huffaker, A., Sims, J. W., Christensen, S. A., Lu, X., Okada, K., \& Peters, R. J. (2014). Biosynthesis: elicitation and roles of monocot terpenoid phytoalexins. . The Plant Journal, 79(4), 659-678. doi:10.1111/tpj.12436

Sieg, R. D., \& Kubanek, J. (2013). Chemical ecology of marine angiosperms: opportunities at the interface of marine and terrestrial systems. Journal of Chemical Ecology, 39(6), 687-711. doi:10.1007/s10886-013-0297-9

Steele, L. T., Caldwell, M., Boettcher, A., \& Arnold, T. (2005). Seagrass-pathogen interactions: "pseudo-induction" of turtlegrass phenolics near wasting disease lesions . Marine Ecology Progress Series, 303, 123-131. doi:10.3354/meps303123 
Recibido:

15/marzo/2019

Aceptado:

29/julio/2019
Torre-Núñez, E., Rodeiro-Guerra, I., Menéndez-Soto, I. R., \& Pérez-Carrasco, D. (2012). Thalassia testudinum, una planta marina con potencialidades de uso terapéutico. Revista Cubana de Plantas Medicinales, 17(3), 288-296.

Trevathan-Tackett, S. M., Lane, A. L., Bishop, N., \& Ross, C. (2015). Metabolites derived from the tropical seagrass Thalassia testudinum are bioactive against pathogenic Labyrinthula sp. Aquatic Botany, 122, 1-8. doi:10.1016/j.aquabot.2014.12.005

Ulloa, U. E., Acevedo-Rodríguez, P., Beck, S., Belgrano, M. J., ..., \& Jørgensen, P. M. (2017). An integrates assessment of the vascular plant species of the Americas. Science, 358, 1614-1617.

Vergeer, L. H., \& Develi, A. (1997). Phenolic acids in healthy and infected leaves of Zostera marina and their growth-limiting properties towards Labyrinthula zosterae. Aquatic Botany, 58(1), 65-72. doi:10.1016/S0304-3770(96)01115-1

Vergeer, L. H., Aarts, T. L., \& de Groot, J. D. (1995). The wasting disease and the effect of abiotic factors (light intensity, temperature, salinity) and infection with Labyrinthula zosterae on the phenolic content of Zostera marina shoots. Aquatic Botany, 52, 35-44. doi:10.1016/0304-3770(95)00480

Vergés, A., Becerro, M. A., Alcoverro, T., \& Romero, J. (2007). Experimental evidence of chemical deterrence against multiple herbivores in the seagrass Posidonia oceanica. Marine Ecology Progress Series, 343, 107-114. doi:10.3354/meps06885

Villaseñor , J. L. (2016). Checklist of the native vascular plants of Mexico. Revista Mexicana de Biodiversidad, 87(3), 559-902.

Waterman, P. G., \& Mole, S. (1994). Analysis of phenolic plant metabolites. Ecological methods and concepts (Vol. 1). (WILEY, Ed.)

Yero-Espinosa, L., Rodrígues-Fernández, Y., \& Pérez-Sosa, N. D. (2017). Caracterización fitoquímica y bromatológica de la Thalassia testudinum usada en alimentación de ovinos pelibuey de Cuba (Ovis aries) . Ciencia \& Futuro, 7(3), 25-36.

Zidorn, C. (2016). Secondary metabolites of seagrasses (Alismatales and Potamogetonales; Alismatidae): Chemical diversity, bioactivity, and ecological function. Phytochemistry, 124, 5-28. doi:10.1016/j.phytochem.2016.02.004 